Artículos científicos

\title{
Validación de entrevistas por juicio de expertos en el estudio de la inclusión educativa en el área de lenguas extranjeras
}

Validation of Interviews by Judgment of Experts in the Study of Inclusive Education in the Foreign Languages Area

Validação de entrevistas por julgamento de especialistas no estudo da inclusão educacional na área de línguas estrangeiras

Veronika de la Cruz Villegas

Universidad Juárez Autónoma de Tabasco, México veronika.delacruz@ujat.mx https://orcid.org/0000-0003-3570-2021

Eduardo Jesús Gordillo Fuentes

Universidad Juárez Autónoma de Tabasco, México eduardojesusg@hotmail.com https://orcid.org/0000-0002-0968-6526 


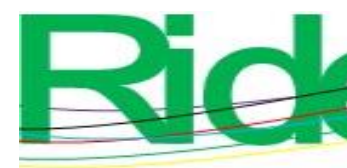

Revista Iberoamericana para la Investigación y el Desarrollo Educativo ISSN $2007-7467$

\section{Resumen}

El objetivo de este artículo es mostrar las experiencias en torno a la conceptualización, validación e implementación de entrevistas elaboradas para analizar las prácticas educativas que convergen en la enseñanza y el aprendizaje de lenguas extranjeras a estudiantes con discapacidad visual en una universidad pública mexicana. Como estrategia de investigación, se diseñaron, validaron (a través del método de consulta a expertos) y aplicaron tres entrevistas para recuperar información sobre las prácticas educativas de carácter institucional, educativo y social que han impactado en los procesos de enseñar y aprender una lengua extranjera en estudiantes con discapacidad visual en el nivel superior. El diseño metodológico consistió de cinco pasos: 1) la elaboración del instrumento, 2) la implementación de este, 3 ) la consulta a expertos, 4) la reelaboración del instrumento y 5) el consenso de los expertos. Los hallazgos muestran que el uso del proceso de validación en cinco pasos secuenciales garantiza, a través del registro de las valoraciones de los jueces, antes y después del pilotaje del instrumento, la organización clara y coherente de la información en ítems de acuerdo con cada dimensión. El análisis de los datos obtenidos durante el proceso de validación arrojó un alto consenso por parte de los expertos, con una alta coherencia interna (alfa de Cronbach > 0.80) en cada una de las secciones de los instrumentos.

Palabras clave: discapacidad visual, entrevistas, inclusión educativa, metodología, validación.

\section{Abstract}

The purpose of this article is to show the experiences around the conceptualization, validation and implementation of interviews developed to analyze the educational practices that converge in second language teaching and learning processes to students with visual impairment in a Mexican public university. As a research strategy, three interviews were designed, validated (through the method of consulting experts) and applied to retrieve information on educational practices of an institutional, educational and social nature that have impacted the processes of teaching and learning a foreign language in students with visual impairment in the upper level. The methodological design consisted of five steps: 1) the elaboration of the instrument, 2) the implementation of the instrument, 3) the consultation 


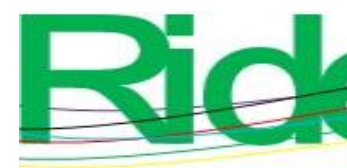

\section{Revista Iberoamericana para la Investigación y el Desarrollo Educativo ISSN 2007 - 7467}

of experts, 4) the re-elaboration of the instrument and 5) the consensus of the experts. Findings show that the use of the validation process in five sequential steps guarantees, through the registration of the judges' opinion, the clear and coherent organization of the information in items according to each dimension. The analysis of data obtained during the validation process showed a high consensus on the part of the experts with a high internal coherence (Cronbach's alpha > 0.80) in each of the sections of the instruments.

Keywords: visual impairment, interviews, inclusive education, method, validation.

\section{Resumo}

O objetivo deste artigo é mostrar as experiências em torno da conceituação, validação e implementação de entrevistas elaboradas para analisar as práticas educacionais que convergem no ensino e aprendizagem de línguas estrangeiras para estudantes com deficiência visual em uma universidade pública mexicana. Como estratégia de pesquisa, foram elaboradas três entrevistas, validadas (através do método de consultoria de especialistas) e aplicadas para recuperar informações sobre práticas educacionais de natureza institucional, educacional e social que impactaram os processos de ensino e aprendizagem de uma língua estrangeira. em alunos com deficiência visual no nível superior. O desenho metodológico consistiu em cinco etapas: 1) a elaboração do instrumento, 2) sua implementação, 3) a consulta de especialistas, 4) a reelaboração do instrumento e 5) o consenso dos especialistas. Os resultados mostram que o uso do processo de validação em cinco etapas seqüenciais garante, por meio do registro das avaliações dos juízes, antes e depois da pilotagem do instrumento, a organização clara e coerente das informações nos itens de acordo com cada dimensão. . A análise dos dados obtidos durante o processo de validação gerou um alto consenso por parte dos especialistas, com alta coerência interna (alfa de Cronbach>0,80) em cada uma das seções do instrumento.

Palavras-chave: deficiência visual, entrevistas, inclusão educacional, metodologia, validação.

Fecha Recepción: Febrero 2020

Fecha Aceptación: Julio 2020 


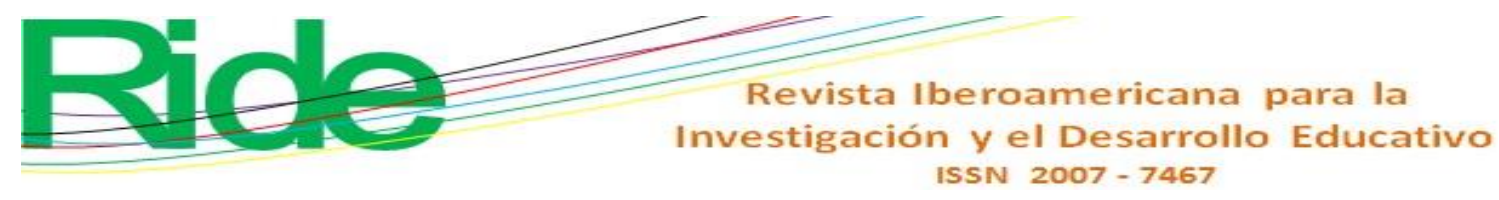

\section{Introducción}

Los motivos, objetivos y alcances de la educación inclusiva han sido cuestionados y debatidos por especialistas en educación durante muchas décadas (Deng y Guo, 2007; Ghergut, 2010; Malinen, Savolainen y Xu, 2012; Mustafa, 2011; Plancarte, 2017; Shevlin, Kenny y Me Neela, 2004; Unianua, 2012). Estos debates inicialmente condujeron al surgimiento de políticas, estrategias, métodos y técnicas que apostaban a la creación de una sociedad justa y digna para niños con necesidades especiales. En la actualidad, desde la Declaración de Incheon para la Educación 2030 (Organización de las Naciones Unidas para la Educación, la Ciencia y la Cultura [Unesco], 2016), existe el siguiente compromiso:

Promover oportunidades de aprendizaje de calidad a lo largo de la vida para todos, en todos los contextos y en todos los niveles educativos. Ello incluye un mayor acceso en condiciones de igualdad a la enseñanza y formación técnica y profesional de calidad, a la educación superior y a la investigación, prestando la debida atención a la garantía de la calidad (p. 8).

A este respecto, la Unesco (2016) considera que garantizar la igualdad de oportunidades para todos en la educación sigue siendo un desafío mundial. Según esta organización, las acciones de educación inclusiva son necesarias para eliminar los obstáculos que se interponen entre la participación y el desempeño de todos los alumnos, y eliminar todas las formas de discriminación en el campo del aprendizaje. Estas acciones deben considerar la diversidad de sus necesidades, capacidades y particularidades (Unesco, 2016).

En México, al igual que en el resto del mundo, la presencia de estudiantes con diversas discapacidades en las universidades es una realidad que no puede ser ignorada. Su formación profesional y preparación para el campo laboral está en manos no solo de los profesores, sino de quienes administran las instituciones de educación y, por supuesto, de ellos mismos. Ver a estos estudiantes transitar por los pasillos de nuestras universidades y asistir a nuestras propias aulas nos ha llevado a reflexionar respecto a las actuales prácticas educativas llevadas a cabo por todos los actores involucrados en su formación profesional, respecto a la efectividad y el impacto de estas.

Por todo lo anterior, en una universidad pública de México se realizó un trabajo de investigación que tuvo como objetivo analizar las prácticas educativas que convergen en la enseñanza y el aprendizaje de lenguas extranjeras a estudiantes con discapacidad visual en el nivel superior. Para este estudio se trabajó en la conceptualización y validación de tres 

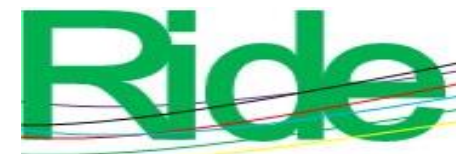

Revista Iberoamericana para la Investigación y el Desarrollo Educativo

ISSN 2007 - 7467

entrevistas dirigidas a los actores directos del proceso educativo, a saber, estudiantes, profesores y personal administrativo de la institución participante, con la intención de obtener datos que permitieran dar respuesta a la siguiente pregunta de investigación: ¿cuáles son las prácticas educativas que desarrolla la institución de educación superior participante en atención a estudiantes con discapacidad visual en el contexto de la enseñanza y el aprendizaje de lenguas extranjeras?

Para llevar a cabo este trabajo se empleó la entrevista como una estrategia para la recolección de datos, la cual es una técnica que, entre sus múltiples ventajas, satisface los requerimientos de interacción personal originados por la civilización (Acevedo y López, 2008).

Cabe mencionar que, con el objetivo de recabar información que permitiera evidenciar las prácticas educativas realizadas por todos los actores involucrados en el proceso educativo enfocado a los estudiantes con discapacidad visual, se diseñaron originalmente dos entrevistas, una para los estudiantes con discapacidad visual (Ginsburg, 1997) y otra para los profesores de lenguas extranjeras.

Cada uno de los instrumentos se piloteó y validó a través del apoyo de profesores especialistas en metodología de la investigación, inclusión educativa y enseñanza de lenguas extranjeras. Como resultado del pilotaje y la validación de los instrumentos, se decidió trabajar tres entrevistas, una para profesores, otra para estudiantes con discapacidad visual y una más para administrativos, debido a que las dos anteriores no eran suficientes para recabar información referente a una de las categorías del estudio (Institucional).

Las siguientes líneas presentan el proceso llevado a cabo desde la conceptualización de las entrevistas hasta la implementación de estas. 


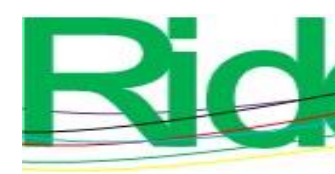

Revista Iberoamericana para la Investigación y el Desarrollo Educativo ISSN $2007-7467$

\section{Materiales y métodos}

La dinámica fugaz de la cotidianidad en las instituciones de educación superior en México nos aleja cada vez más de nuestra realidad, de la reflexión de lo que hacemos y cómo lo hacemos. Esta dinámica está permeando de forma significativa tanto en nuestros canales de comunicación como en los mecanismos de transferencia de saberes: cada vez es más complejo el trabajo áulico y, por ende, el óptimo desarrollo de la educación. Ante este panorama, la realidad se vuelve aún más compleja cuando la razón de ser de nuestras acciones es un estudiante con discapacidad visual, situación que lleva a cuestionarnos sobre las acciones que llevamos a cabo en el aula y el impacto de estas con miras a su formación integral.

A partir de un contexto en específico como el de las lenguas extranjeras, y con el objetivo de recabar información que permitiera evidenciar las prácticas de carácter educativo, social e institucional realizadas tanto por profesores como por estudiantes y coordinadores que impactan en la enseñanza y el aprendizaje de lenguas extranjeras a estudiantes con discapacidad visual, se diseñaron originalmente dos entrevistas, una para los estudiantes con discapacidad visual y otra para los profesores de lenguas extranjeras (Ginsburg, 1997). A través de las siguientes líneas se detalla el proceso metodológico llevado a cabo para la validación de las entrevistas.

\section{Entrevista al profesor de lenguas extranjeras}

Para el caso de la entrevista al profesor de lenguas extranjeras, se llevó a cabo una doble valoración enmarcada en un proceso de cinco pasos (ver figura 1). El primer paso consistió en la conceptualización de la entrevista, la cual se aplicó a un sujeto con características similares a las de los verdaderos sujetos de estudio (paso dos). El paso tres se caracterizó por el acercamiento hacia con los expertos, a quienes les fue presentado el instrumento, el audio (producto de la prueba piloto) y la descripción general del estudio para el que había sido aplicado. El paso cuatro consistió en la reconceptualización del instrumento a partir de las recomendaciones proporcionadas por el grupo de expertos. Y finalmente, se presentó la versión final del instrumento a los expertos para su aprobación. 


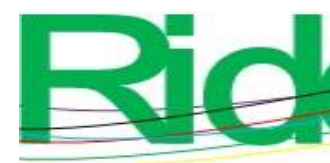

\section{Revista Iberoamericana para la Investigación y el Desarrollo Educativo ISSN $2007-7467$}

Figura 1. Proceso metodológico para la validación de la entrevista

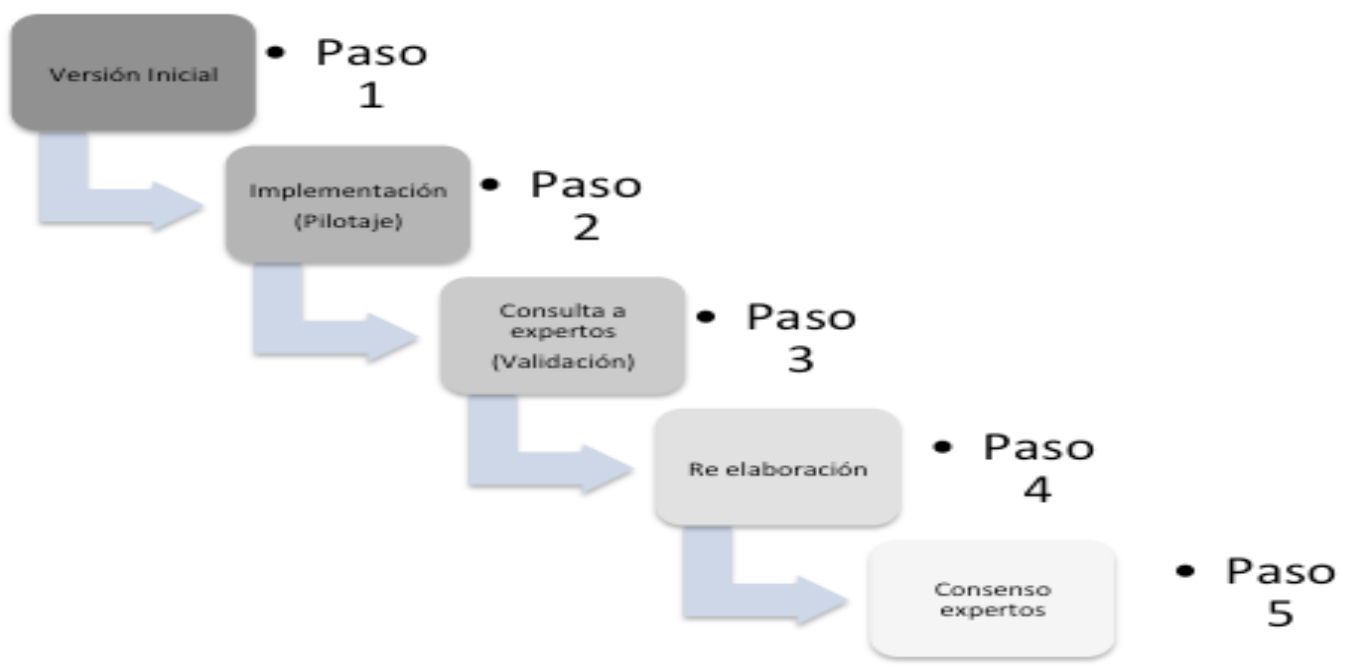

Fuente: Elaboración propia

\section{Paso uno. Versión inicial}

En cuanto a la conceptualización de la entrevista, recabar información que diera cuenta de las prácticas llevadas a cabo por diversos actores en relación con tres categorías, a saber, Educativo, Institucional y Social, en el contexto del aula de lenguas extranjeras, implicó remontar a estudiantes, profesores y administrativos a tiempos, espacios y situaciones en particular de su experiencia educativa. Por tal motivo se revisaron diversos procesos de validación de expertos, entre las que destacamos la de Galicia, Balderrama y Edel (2017) y la experiencia de Dorantes, Hernández y Tobón (2016), y guías de entrevista, entre las que sobresalió la de Ginsburg (1997). La entrevista clínica de Ginsburg (1997) nos proporcionó una guía sobre la estructura de los ítems, lo cual permitió contextualizar al sujeto de estudio ubicándolo en tiempo y espacio.

En un primer momento, se elaboró la entrevista del profesor de lenguas extranjeras, previa selección de los temas y constructos, la cual poseía la estructura que se muestra en la tabla 1. Con base en una matriz de coherencia, se elaboró otra matriz que permitió organizar las preguntas del instrumento, teniendo en cuenta las categorías y dimensiones del estudio. 
Tabla 1. Visión gráfica de la entrevista inicial para profesores de lenguas extranjeras que atienden o han atendido a estudiantes con discapacidad visual

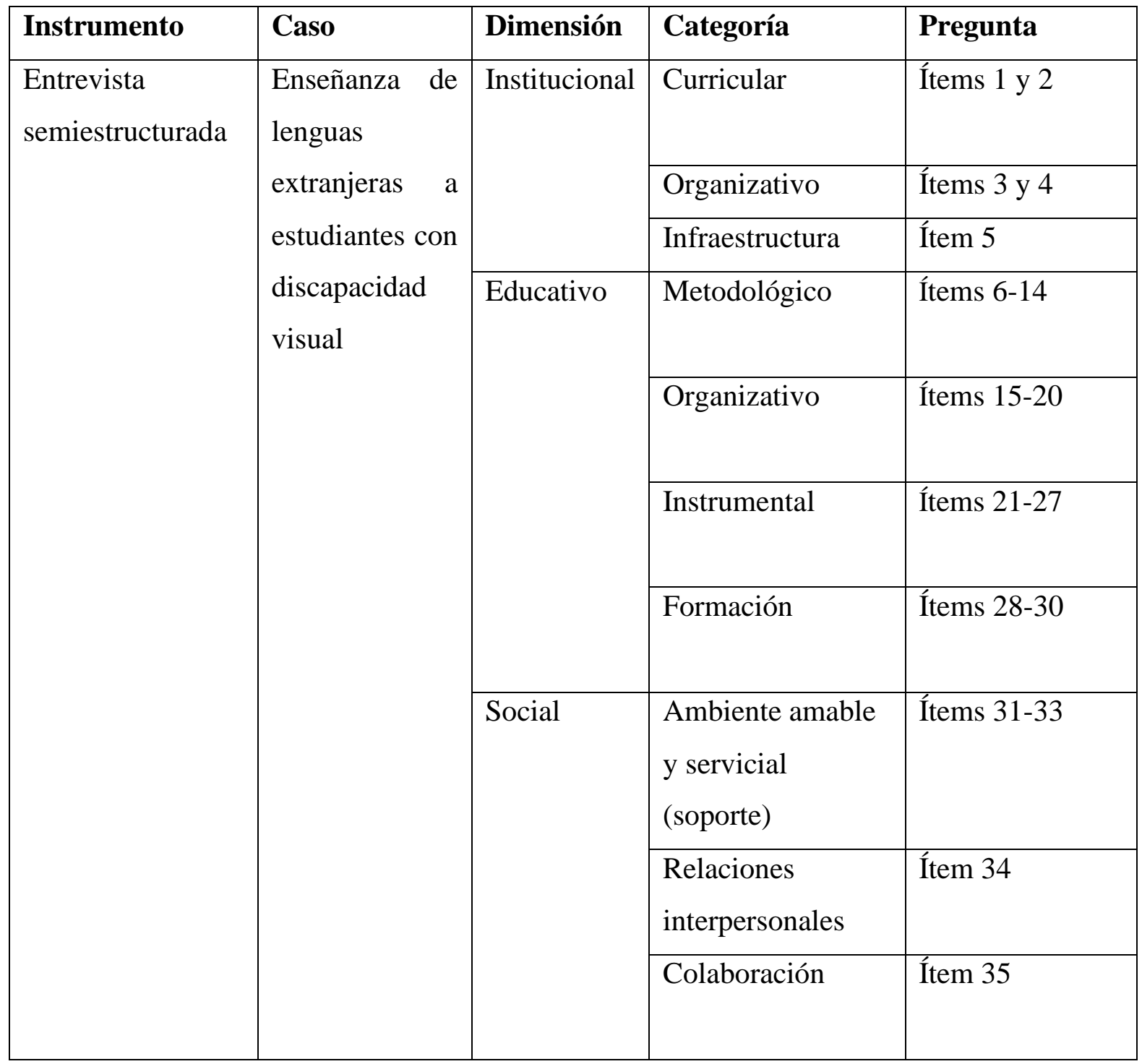

Fuente: Elaboración propia

Un total de 35 preguntas conformaban la guía de entrevista, las cuales se encontraban agrupadas en tres dimensiones (Institucional, Educativo y Social). La primera y tercera dimensión contaban con cinco preguntas que buscaban identificar información relativa a los aportes institucionales en los procesos de inclusión de estudiantes con discapacidad visual y a las relaciones interpersonales dadas entre estudiantes-estudiante con discapacidad visual. La segunda categoría contaba con 25 preguntas, lo cual la ubicaba como la sección más amplia de la entrevista. La intención aquí era obtener información específica sobre cuestiones didácticas, curriculares, organizativas y de formación docente. Cada una de las dimensiones, 


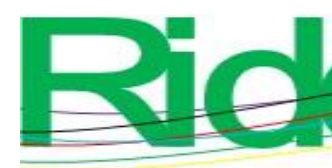

Revista Iberoamericana para la Investigación y el Desarrollo Educativo

ISSN 2007 - 7467

a su vez, contaba con una presentación: el planteamiento de una situación que buscaba ubicar al profesor en un momento y espacio específicos de su experiencia, lo que le permitiría recordar los aspectos que le serían cuestionados.

\section{Paso dos. Implementación (pilotaje)}

Se aplicó la entrevista a una profesora de italiano con experiencia en atención a estudiantes con discapacidad visual en el nivel superior, cuyo audio fue grabado. Una vez realizada esta, se entregaron ambos elementos (guía de entrevista y audio), más la descripción general del estudio (título, objetivos, preguntas de investigación y supuestos), a cinco expertos para su valoración.

\section{Paso tres. Consulta a expertos (validación)}

La selección de los expertos se realizó teniendo como base las siguientes características: 1) conocimiento sobre la enseñanza de lenguas extranjeras, 2) experiencia en inclusión educativa y 3) conocimiento de la metodología de la investigación.

Tabla 2. Características del grupo de expertos seleccionados para la investigación

\begin{tabular}{|c|c|c|}
\hline Formación & Especialidad & Experiencia \\
\hline Doctorado en Lingüística Aplicada & Enseñanza del inglés & 18 años \\
\hline Licenciatura en Idiomas & Enseñanza del inglés & 15 años \\
& Inclusión educativa & 8 años \\
\hline Licenciatura en Idiomas & Enseñanza del inglés & \\
\hline Maestría en Docencia & Inclusión educativa & 12 años \\
\hline Doctorado en Educación & Inclusión educativa & 10 años \\
\hline
\end{tabular}

Fuente: Elaboración propia

Los expertos fueron contactados vía telefónica y la información les fue enviada por correo electrónico. Las sesiones para la validación de los instrumentos se llevaron a cabo en las instalaciones de la institución participante, y en el caso específico de un experto de otro estado de la república mexicana, se trabajó a través de videoconferencia.

El proceso de valoración fue el siguiente. Se consideró pertinente llevar el instrumento a su aplicación para verificar su correcto diseño. Para tal efecto, se emplearon 

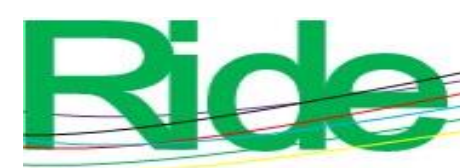

Revista Iberoamericana para la Investigación y el Desarrollo Educativo

ISSN 2007 - 7467

dos tipos de valoraciones: la primera buscó probar la claridad expositiva de las frases (de contenido) y la segunda, la eficacia de los datos (eficacia del contenido) para poder efectuar con ellos un análisis que permitiera identificar las prácticas educativas que profesores, estudiantes con discapacidad visual y personal administrativo realizan para favorecer la enseñanza y el aprendizaje de las lenguas extranjeras entre estudiantes con discapacidad visual. Para los dos tipos de valoración se consideró tomar una muestra no representativa, una muestra por cada instrumento.

Con el objetivo de lograr la validación del contenido de los ítems, se diseñó un instrumento con siete situaciones y los 35 ítems de la entrevista, a los que se les colocó, para la valoración de los cinco expertos, la siguiente escala de Likert: el valor 1 correspondía a inaceptable; el valor 2 a deficiente; 3 a regular; 4 a aceptable, y 5 correspondía a eficiente. En un primer momento, se trabajó en la valoración de las situaciones y posteriormente en la valoración de los ítems.

Con respecto a las situaciones que se plantean al inicio de un grupo de preguntas, el primer experto (enseñanza de lenguas extranjeras) valoró seis situaciones como eficientes y una como regular. El segundo experto (profesora de lenguas extranjeras con experiencia en atención a estudiantes con discapacidad visual) valoró seis situaciones como eficientes y una como deficiente y recomendó trabajar en la redacción de uno de los ítems. El tercer experto valoró cinco situaciones como aceptables y una como regular y recomendó eliminar una situación. El cuarto experto valoró las seis situaciones entre eficientes y aceptables. Y el quinto experto valoró cuatro situaciones como aceptables, una regular y una como deficiente (tabla 3). 


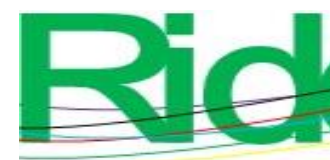

Revista Iberoamericana para la Investigación y el Desarrollo Educativo ISSN 2007 - 7467

Tabla 3. Valoración del contenido de la entrevista del profesor

\begin{tabular}{|c|c|c|c|c|c|c|c|c|c|}
\hline \multirow{2}{*}{$\begin{array}{c}\text { Núm. } \\
\text { de } \\
\text { formul } \\
\text { ario }\end{array}$} & \multicolumn{7}{|c|}{ Situaciones } & \multirow{2}{*}{$\begin{array}{l}\text { Puntaj } \\
\text { e total }\end{array}$} & \multirow[t]{2}{*}{ Comentarios } \\
\hline & 1 & 2 & 3 & 4 & 5 & 6 & 7 & & \\
\hline 1 & $E$ & $E$ & $E$ & $E$ & $\mathrm{E}$ & $\mathrm{R}$ & $E$ & 33 & $\begin{array}{l}\text { La sección sobre lo institucional debe } \\
\text { ser explorada a través de otros sujetos } \\
\text { (director, administrador). }\end{array}$ \\
\hline 2 & $\mathrm{E}$ & $\mathrm{E}$ & $\mathrm{E}$ & $\mathrm{E}$ & $\mathrm{E}$ & $\mathrm{D}$ & $\mathrm{E}$ & 32 & $\begin{array}{l}\text { Reducir las situaciones, ya que } \\
\text { parece que se repiten las ideas. }\end{array}$ \\
\hline 3 & $\mathrm{~A}$ & $\mathrm{~A}$ & $\mathrm{~A}$ & $\mathrm{~A}$ & A & $\mathrm{R}$ & $\mathrm{A}$ & 27 & $\begin{array}{l}\text { Eliminar una situación. No hay } \\
\text { diferencia entre una y otra. } \\
\text { Recomiendo fusionar las que son } \\
\text { parecidas. }\end{array}$ \\
\hline 4 & $\mathrm{~A}$ & $\mathrm{E}$ & $E$ & $\mathrm{~A}$ & A & $E$ & $\mathrm{~A}$ & 31 & $\begin{array}{l}\text { Podría utilizarse una misma situación } \\
\text { para dos aspectos, como en el caso de } \\
\text { lo administrativo e instrumental. }\end{array}$ \\
\hline 5 & $\mathrm{~A}$ & $\mathrm{~A}$ & $\mathrm{R}$ & $\mathrm{A}$ & $\mathrm{A}$ & $\mathrm{D}$ & $\mathrm{A}$ & 25 & $\begin{array}{l}\text { Trabajar en la redacción de la } \\
\text { primera situación. No recomiendo } \\
\text { incluir el término apoyo entre } \\
\text { paréntesis. }\end{array}$ \\
\hline
\end{tabular}

Fuente: Elaboración propia

Así pues, en su mayoría, las situaciones fueron valoradas de manera positiva. En lo que se refiere a las situaciones marcadas con menor puntaje, los expertos coincidieron en que estas presentaban algunas deficiencias, para lo que recomendaron reducir algunas de ellas, como en el caso de la situación cinco, además de agrupar aquellas actividades que coincidieran con la lógica de alguna de las otras situaciones, ya que les parecían un tanto repetitivas. Igualmente, se hizo la recomendación de trabajar en la redacción de algunas de las situaciones y evitar ser tan repetitivos con algunos términos. Para finalizar, se elaboró una nueva revisión del instrumento y se realizaron los ajustes pertinentes. 


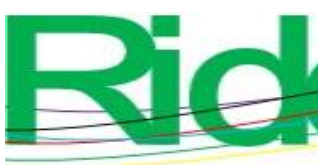

Revista Iberoamericana para la Investigación y el Desarrollo Educativo

ISSN $2007-7467$

Con respecto a las 35 preguntas relacionadas con las categorías de lo Institucional, Educativo y Social, el primer experto las valoró entre eficientes y aceptables y recomendó revisar la redacción de algunos ítems. El segundo experto valoró el contenido de los ítems entre aceptable, eficiente, regular y deficiente, y recomendó realizar preguntas más específicas y evitar con ello la saturación de algunos ítems. El tercer experto valoró el contenido entre aceptable, eficiente y regular. La valoración del cuarto experto coincidió en su mayoría con la valoración del segundo experto, con valores entre lo aceptable, eficiente, regular y deficiente. El quinto experto valoró las 35 preguntas entre eficientes y aceptables y recomendó trabajar en la redacción de algunas de ellas, principalmente recomendó evitar la repetición (ver tabla 4)

Tabla 4. Validación del contenido de la entrevista del profesor

\begin{tabular}{|c|c|c|c|c|c|c|c|c|c|c|c|c|c|c|c|c|c|c|c|c|c|c|c|c|c|c|c|c|c|c|c|c|c|c|c|c|c|}
\hline \multirow[t]{2}{*}{ \# } & \multicolumn{36}{|c|}{ Preguntas } & Total \\
\hline & 1 & 2 & 3 & 4 & 5 & 6 & 7 & 8 & \begin{tabular}{l|}
9 \\
\end{tabular} & 0 & \begin{tabular}{l|} 
\\
1 \\
\end{tabular} & $\begin{array}{l}1 \\
2\end{array}$ & 3 & \begin{tabular}{l|} 
\\
4
\end{tabular} & $\begin{array}{l}1 \\
5\end{array}$ & $\begin{array}{l}1 \\
6\end{array}$ & $\begin{array}{l}1 \\
7\end{array}$ & $\begin{array}{l}1 \\
8\end{array}$ & $\begin{array}{l}1 \\
9\end{array}$ & $\begin{array}{l}2 \\
0\end{array}$ & $\begin{array}{l}2 \\
1\end{array}$ & $\begin{array}{l}2 \\
2\end{array}$ & $\begin{array}{l}2 \\
3\end{array}$ & $\begin{array}{l}2 \\
4\end{array}$ & \begin{tabular}{|l|}
2 \\
5
\end{tabular} & $\begin{array}{l}2 \\
6\end{array}$ & $\begin{array}{l}2 \\
7\end{array}$ & $\begin{array}{l}2 \\
8\end{array}$ & $\begin{array}{l}2 \\
9\end{array}$ & $\begin{array}{l}3 \\
0\end{array}$ & & & \begin{tabular}{l|}
3 \\
2 \\
2
\end{tabular} & $\begin{array}{l}3 \\
3\end{array}$ & & & \\
\hline 1 & $\mathrm{~A}$ & $\mathrm{~A}$ & $\mathrm{E}$ & $\mathrm{E}$ & $E$ & $\mathrm{E}$ & $\mathrm{E}$ & $\mathrm{E}$ & $\mathrm{E}$ & $\mathrm{E}$ & $\mathrm{A}$ & $\mathrm{A}$ & $\mathrm{A}$ & $E$ & $\mathrm{E}$ & $\mathrm{E}$ & $\mathrm{A}$ & $\mathrm{A}$ & $\mathrm{A}$ & $\mathrm{A}$ & $\mathrm{A}$ & $\mathrm{E}$ & $E$ & $\mathrm{~A}$ & $\mathrm{~A}$ & E & $\mathrm{E}$ & E & $\bar{F}$ & $\bar{E}$ & E & $\bar{E}$ & $\mathrm{E}$ & $\mathrm{A}$ & $\mathrm{A}$ & $\mathrm{A}$ & 155 \\
\hline 2 & $\mathrm{E}$ & $\mathrm{E}$ & $\mathrm{R}$ & $\mathrm{D}$ & $\mathrm{D}$ & $\mathrm{E}$ & $\mathrm{R}$ & $\mathrm{E}$ & $\mathrm{E}$ & $\bar{E}$ & $\mathrm{~A}$ & $\mathrm{~A}$ & $\mathrm{~A}$ & $\mathrm{R}$ & \begin{tabular}{l|l}
$R$ \\
\end{tabular} & $\mathrm{R}$ & $\mathrm{A}$ & $\mathrm{A}$ & $\mathrm{A}$ & $\mathrm{A}$ & $\mathrm{A}$ & $\mathrm{E}$ & \begin{tabular}{|c|}
$E$ \\
\end{tabular} & $\mathrm{~A}$ & $\mathrm{~A}$ & $\mathrm{R}$ & $\mathrm{R}$ & $\bar{R}$ & $\bar{F}$ & $\bar{R}$ & R & $\mathrm{R}$ & $\mathrm{R}$ & $\mathrm{A}$ & $\mathrm{A}$ & $\mathrm{A}$ & \begin{tabular}{|l|}
137 \\
\end{tabular} \\
\hline 3 & $\mathrm{~A}$ & $\mathrm{~A}$ & $\mathrm{R}$ & $\mathrm{E}$ & A & $\mathrm{A}$ & $\mathrm{A}$ & $\mathrm{A}$ & $\mathrm{A}$ & $\mathrm{A}$ & $\mathrm{A}$ & $\mathrm{A}$ & $\mathrm{R}$ & $\mathrm{R}$ & $\mathrm{A}$ & $\mathrm{A}$ & $\mathrm{A}$ & $\mathrm{A}$ & $\mathrm{A}$ & $\mathrm{R}$ & $\mathrm{E}$ & $\mathrm{A}$ & $\mathrm{R}$ & $\mathrm{E}$ & $\mathrm{E}$ & $\mathrm{R}$ & $\mathrm{R}$ & $\bar{R}$ & $\mathrm{~F}$ & $\vec{A}$ & & A & $\mathrm{E}$ & $\mathrm{A}$ & $\mathrm{A}$ & $\mathrm{A}$ & \begin{tabular}{|l|l|}
152 \\
\end{tabular} \\
\hline 4 & $\mathrm{~A}$ & $E$ & $\mathrm{R}$ & $\mathrm{R}$ & $\mathrm{D}$ & $\mathrm{E}$ & $\mathrm{R}$ & $\mathrm{E}$ & $\mathrm{E}$ & $\mathrm{E}$ & $\mathrm{A}$ & $\mathrm{A}$ & $\mathrm{A}$ & $\mathrm{R}$ & $\mathrm{R}$ & $\mathrm{R}$ & $\mathrm{A}$ & $\mathrm{A}$ & $\mathrm{A}$ & $\mathrm{A}$ & $\mathrm{A}$ & $\mathrm{E}$ & $\mathrm{E}$ & $\mathrm{A}$ & A & $\mathrm{E}$ & $\mathrm{R}$ & $\mathrm{R}$ & F & R & & $\mathrm{R}$ & $\mathrm{R}$ & $\mathrm{A}$ & $\mathrm{A}$ & $\mathrm{A}$ & \begin{tabular}{|l|l|}
140 \\
\end{tabular} \\
\hline 5 & $\mathrm{E}$ & $\mathrm{A}$ & $\mathrm{E}$ & $\mathrm{A}$ & $\mathrm{A}$ & $\mathrm{A}$ & $\mathrm{A}$ & $\mathrm{A}$ & $\mathrm{A}$ & $\mathrm{A}$ & $\mathrm{A}$ & $\mathrm{A}$ & $\mathrm{A}$ & $\mathrm{A}$ & $\mathrm{A}$ & $\mathrm{A}$ & $\mathrm{A}$ & $\mathrm{A}$ & $\mathrm{A}$ & $\mathrm{A}$ & $\mathrm{E}$ & $\mathrm{A}$ & $\mathrm{E}$ & $\mathrm{E}$ & $E$ & $E$ & $\mathrm{E}$ & E & $\overline{\mathrm{F}}$ & $\bar{E}$ & & $\bar{E}$ & $\mathrm{~A}$ & $\mathrm{~A}$ & $\mathrm{~A}$ & $\mathrm{~A}$ & \begin{tabular}{|l|l}
163 \\
\end{tabular} \\
\hline
\end{tabular}

Fuente: Elaboración propia

Teniendo en cuenta el conjunto de recomendaciones realizadas por los expertos, se revisó nuevamente el instrumento y se realizaron los ajustes pertinentes; entre ellos resalta el descarte de algunos ítems y el reordenamiento de estos, con base en una secuencia lógica, independientemente de la categoría a la que pertenecieran.

Con el objetivo de lograr la validación de la eficacia del contenido de los ítems, se diseñó un instrumento con los 35 ítems de la entrevista, a los que se les colocó, para la valoración de los cinco expertos, una nueva escala de Likert, donde 1 indicaba totalmente en desacuerdo; 2, en desacuerdo; 3, neutro; 4, de acuerdo y 5 totalmente de acuerdo. La característica principal de este instrumento es que presentaba el objetivo de cada ítem, la categoría a la que pertenecía y el indicador correspondiente. Por ejemplo, el ítem número 23, correspondiente a la categoría Educativo, con indicador Organizativo, tiene como objetivo conocer la forma en la que el profesor emplea los recursos tecnológicos en el aula y plantea lo siguiente: “Describa cómo utilizó esos recursos en el aula". Los expertos valoraron la 


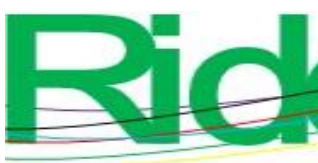

\section{Revista Iberoamericana para la
Investigación y el Desarrollo Educativo ISSN 2007 - 7467}

eficacia del contenido de la siguiente manera: los cinco expertos coincidieron en valorar la mayoría de los ítems con el mayor puntaje; los menores puntajes estuvieron dirigidos a los ítems correspondientes a las categorías Institucional y Metodológico, específicamente en el indicador Organizativo (ver tabla 5).

Tabla 5. Validación de la eficacia del contenido de la entrevista del profesor

\begin{tabular}{|c|c|c|c|c|c|c|c|c|c|c|c|c|c|c|c|c|c|c|c|c|c|c|c|c|c|c|c|c|c|c|c|c|c|c|c|c|c|}
\hline \multirow[t]{3}{*}{ \# } & \multicolumn{36}{|c|}{ Preguntas } & Total \\
\hline & 1 & 2 & 3 & \begin{tabular}{|l|l}
4 \\
\end{tabular} & 5 & 6 & 7 & 8 & 9 & 1 & 1 & 1 & 1 & 1 & 1 & 1 & 1 & 1 & & 2 & 2 & 2 & 2 & 2 & 2 & 2 & 2 & 2 & 2 & 3 & 3 & & 3 & & 3 & 3 & \\
\hline & & & & & & & & & & 0 & 1 & 2 & 3 & 4 & 5 & 6 & 7 & 8 & 9 & 0 & 1 & 2 & 3 & 4 & 5 & 6 & 7 & 8 & 9 & 0 & 1 & & 2 & 3 & 4 & 5 & \\
\hline 1 & 3 & 5 & 1 & 1 & 1 & 4 & 5 & 5 & 5 & 5 & 5 & 5 & 5 & \begin{tabular}{|l|}
5 \\
\end{tabular} & 5 & 5 & \begin{tabular}{|l|}
5 \\
\end{tabular} & 3 & 5 & 5 & 5 & 5 & 5 & 2 & 2 & 3 & 4 & 5 & 4 & 4 & 5 & & 5 & \begin{tabular}{l|}
5 \\
\end{tabular} & 5 & 5 & 148 \\
\hline 2 & 3 & 5 & 2 & 2 & 1 & 5 & 5 & 5 & 5 & 5 & 5 & 5 & 5 & 5 & 5 & 5 & 5 & 4 & 5 & 5 & 5 & 5 & 5 & 3 & 4 & 3 & 5 & 5 & 4 & 4 & 5 & & 5 & \begin{tabular}{l|}
5 \\
\end{tabular} & 5 & 5 & 156 \\
\hline 3 & 4 & 4 & 1 & 2 & 1 & 5 & 5 & 5 & 5 & 5 & 5 & 5 & 5 & 5 & 5 & 5 & 4 & 4 & 5 & 5 & 5 & 5 & 5 & 4 & 4 & 4 & 5 & 5 & 4 & 4 & 5 & & 5 & 5 & 5 & 5 & 156 \\
\hline & & & & & & & & & & & & & & & & & & & & & & & & & & & & & & & & & & & & & \\
\hline 4 & 4 & 4 & 1 & 2 & 1 & 4 & 5 & 5 & 5 & 5 & 5 & 5 & 5 & 5 & 5 & 5 & 4 & 4 & 5 & 5 & 5 & 5 & 5 & 4 & 3 & 4 & 5 & 5 & 5 & 5 & 5 & & 5 & 5 & 5 & 5 & 156 \\
\hline 5 & 3 & 3 & 1 & 2 & 1 & 5 & 5 & 5 & 5 & 5 & 5 & 5 & 5 & 5 & 5 & 5 & 3 & 4 & 5 & 5 & 5 & 5 & 5 & 4 & 4 & 4 & 5 & 5 & 4 & 4 & 5 & & 5 & 5 & 5 & 5 & 155 \\
\hline
\end{tabular}

Fuente: Elaboración propia

Con base en el conjunto de recomendaciones realizadas por los expertos, se revisó nuevamente el instrumento y se realizaron los ajustes pertinentes; aquí resalta, una vez más, la realización de nuevos ítems, el reordenamiento de estos con base en una secuencia lógica, independientemente de la categoría a la que pertenecieran, y la elaboración de un nuevo instrumento enfocado al aspecto Institucional y dirigido específicamente a los administrativos y directivos de la institución de educación superior.

\section{Paso cuatro. Reelaboración}

La estructura que se presenta a continuación se logró a través del proceso de pilotaje y validación por expertos, que permitió enriquecer la estructura y los contenidos iniciales de este instrumento.

La entrevista del profesor de lenguas extranjeras consta de una presentación que incluye el objetivo de la entrevista y los datos generales del profesor entrevistado. La sección de preguntas consta de tres situaciones que tenían como objetivo remontar al profesor de lenguas extranjeras a un momento y situación específicos y 26 ítems distribuidos entre las situaciones antes mencionadas. Cada una de estas situaciones incluye preguntas relacionadas con dos de las tres categorías que desarrolla la entrevista (Educativo y Social). La primera situación plantea lo siguiente: "Pensemos en aquel momento en el que planeaba su clase de lenguas extranjeras para el grupo en el que se encontraba un estudiante con discapacidad 


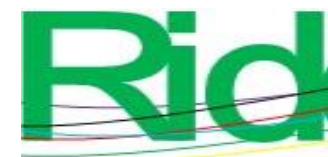

Revista Iberoamericana para la Investigación y el Desarrollo Educativo ISSN 2007 - 7467

visual". Mediante esta situación se buscaba obtener información relacionada con los momentos de planeación y organización del curso. Las cuatro preguntas que se contemplan en esta situación pertenecían a la categoría Educativo.

La segunda situación que se consideró fue la siguiente: "Pensemos en las primeras clases de lengua extranjera en las que contó con la presencia de un estudiante con discapacidad visual". A través de las 16 preguntas que conforman este apartado, se buscaba ubicar al profesor en el momento en el que tenía su primer contacto tanto con el estudiante con discapacidad visual como con sus pares y el aula de clase.

La última situación que se planteó en esta entrevista fue la siguiente: "Pensemos en aquellas sesiones en las que desarrollaba usted alguna actividad grupal con sus estudiantes de lengua extranjera". Esta situación específicamente presenta seis ítems relacionados con la categoría de lo Social, a través de la cual se busca obtener información sobre las relaciones interpersonales entre el profesor y el estudiante con discapacidad visual y este, a su vez, con sus pares. Para finalizar, se presenta el agradecimiento por la participación en la entrevista y el proyecto.

\section{Paso cinco. Consenso con expertos}

El comité de expertos fue convocado nuevamente para la validación de los cambios efectuados sobre la versión inicial del instrumento y emitió resultados favorables.

\section{Entrevista del estudiante con discapacidad visual}

La entrevista del estudiante con discapacidad visual tuvo el mismo tratamiento que el dado a la entrevista con el profesor de lenguas extranjeras, con una doble valoración (contenido y eficacia de contenido).

Se aplicó la entrevista a una estudiante de inglés con discapacidad visual de la Universidad Juárez Autónoma de Tabasco, se grabó el audio y se entregaron ambos elementos (guía de entrevista y audio), además de la descripción general del estudio a cinco expertos para su valoración en el mismo mes. 


\section{Validación del contenido del instrumento}

Para la validación del contenido de los ítems se diseñó un instrumento con las siete situaciones y los 35 ítems de la entrevista, y se adjuntó, para la valoración de los cinco expertos, la escala de Likert puesta a continuación: $1=$ inaceptable; $2=$ deficiente; $3=$ regular; $4=$ aceptable; $5=$ eficiente. Con respecto a las situaciones que se plantean al inicio de un grupo de preguntas, el primer experto valoró las siete situaciones como eficientes y recomendó trabajar en la redacción de los ítems aunque respetando su contenido. El segundo experto (profesora de lenguas extranjeras con experiencia en atención a estudiantes con discapacidad visual) valoró cinco situaciones como eficientes y dos como deficientes y recomendó trabajar en la redacción de estas buscando mayor especificidad contextual. El tercer experto valoró cuatro situaciones como aceptables, una como eficiente y dos como regulares, y recomendó eliminar una situación, ya que consideró que dos referían al mismo contexto. El cuarto experto valoró cinco situaciones como aceptables y dos como eficientes, y emitió la misma recomendación que el evaluador anterior. Por último, el quinto experto valoró tres situaciones como aceptables, dos como eficientes, una como regular y otra como deficiente (tabla 6). 


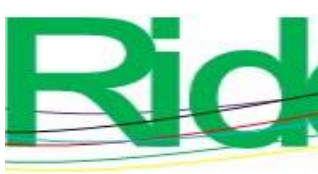

Revista Iberoamericana para la Investigación y el Desarrollo Educativo

ISSN 2007 - 7467

Tabla 6. Validación del contenido de la entrevista del estudiante con discapacidad visual

\begin{tabular}{|c|c|c|c|c|c|c|c|c|c|}
\hline \# & \multicolumn{5}{|c|}{ Situaciones } & \multicolumn{1}{c|}{$\begin{array}{c}\text { Puntaje } \\
\text { total }\end{array}$} & \multicolumn{1}{c}{ Comentarios } \\
\cline { 2 - 7 } & 1 & 2 & 3 & 4 & 5 & 6 & 7 & & \\
\hline 1 & E & E & E & E & E & E & E & 35 & $\begin{array}{l}\text { Se recomienda trabajar en la redacción } \\
\text { de los ítems conservando su contenido. } \\
\text { Insisto en que esta categoría debe estar } \\
\text { sujeta en otro sujetos. Cambiar el orden } \\
\text { de ítems. }\end{array}$ \\
\hline 2 & E & E & D & E & E & D & E & 39 & $\begin{array}{l}\text { Algunas situaciones parecen demasiado } \\
\text { generales. Hay que ser más específico en } \\
\text { su redacción. }\end{array}$ \\
\hline 3 & A & A & E & A & R & R & A & 27 & $\begin{array}{l}\text { Las dos situaciones marcadas con } R \text { son } \\
\text { muy parecidas en cuanto al contexto que } \\
\text { en ellas se plantea. Recomiendo eliminar } \\
\text { una de ellas y agrupar las preguntas. }\end{array}$ \\
\hline 4 & A & A & E & A & A & E & A & 30 & $\begin{array}{l}\text { Hay situaciones que se repiten y podría } \\
\text { utilizarse solo una. }\end{array}$ \\
\hline 5 & A & E & R & A & E & D & A & 27 & $\begin{array}{l}\text { Es importante trabajar en la redacción de } \\
\text { los ítems, tal como se sugiere en el } \\
\text { impreso. }\end{array}$ \\
\hline
\end{tabular}

Fuente: Elaboración propia

Una vez obtenida la valoración del contenido de la entrevista, se observó que, en su mayoría, los ítems fueron valorados de manera positiva. En lo que se refiere a los ítems marcados con menor puntaje, los expertos coincidieron en que estos presentaban algunas deficiencias, para lo que recomendaron reducir algunas situaciones, como en el caso del ítem 5, además de agrupar aquellas actividades que coincidieran con la lógica de alguna de las otras situaciones, ya que les parecían un tanto repetitivas. Igualmente, se hizo la recomendación de trabajar en la redacción de algunas de las situaciones y evitar ser tan repetitivos con algunos términos. Finalmente, se revisó nuevamente el instrumento y se realizaron los ajustes pertinentes.

En cuanto al contenido de las 35 preguntas relacionadas con las categorías Institucional, Educativo y Social, el primer experto las valoró entre aceptables, eficientes y regulares, y recomendó eliminar algunos ítems, principalmente aquellos relacionados con la categoría Institucional. El segundo experto valoró el contenido de los ítems entre aceptable, eficiente, regular y deficiente, y recomendó realizar más preguntas para que las existentes no tuvieran tanta carga de información, lo que pudiera confundir al estudiante. El tercer experto valoró el contenido entre aceptable, eficiente y regular; en este caso, la recomendación fue 


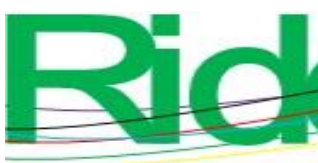

\section{Revista Iberoamericana para la
Investigación y el Desarrollo Educativo ISSN 2007 - 7467}

trabajar en la redacción de los ítems actuales. La valoración del cuarto experto coincidió con la valoración del tercer experto, con valores entre lo aceptable, eficiente y regular, y recomendó lo mismo que el anterior experto. Finalmente, el quinto experto valoró las 35 preguntas entre aceptables, eficientes y regulares, y aconsejó trabajar en la redacción de algunas de ellas y evitar la repetición (véase tabla 7).

Tabla 7. Validación del contenido de la entrevista del estudiante con discapacidad visual

\begin{tabular}{|c|c|c|c|c|c|c|c|c|c|c|c|c|c|c|c|c|c|c|c|c|c|c|c|c|c|c|c|c|c|c|c|c|c|c|c|c|c|}
\hline \# & \multicolumn{36}{|c|}{ Preguntas } & \multirow[t]{2}{*}{ Total } \\
\hline & 1 & 2 & 3 & 4 & 5 & 6 & 7 & 8 & 9 & $\begin{array}{l}1 \\
0\end{array}$ & $\begin{array}{l}1 \\
1\end{array}$ & $\begin{array}{l}1 \\
2\end{array}$ & $\begin{array}{l}1 \\
3\end{array}$ & $\begin{array}{l}1 \\
4\end{array}$ & \begin{tabular}{l|}
1 \\
5
\end{tabular} & \begin{tabular}{l|l}
1 \\
6
\end{tabular} & $\begin{array}{l}1 \\
7\end{array}$ & \begin{tabular}{l|l}
1 \\
8
\end{tabular} & \begin{tabular}{l|}
1 \\
9
\end{tabular} & $\begin{array}{l}2 \\
0\end{array}$ & $\begin{array}{l}2 \\
1\end{array}$ & $\begin{array}{l}2 \\
2\end{array}$ & $\begin{array}{l}2 \\
3\end{array}$ & $\begin{array}{l}2 \\
4\end{array}$ & & & $\begin{array}{l}2 \\
6\end{array}$ & \begin{tabular}{l|}
2 \\
7
\end{tabular} & $\begin{array}{l}2 \\
8\end{array}$ & $\begin{array}{l}2 \\
9\end{array}$ & $\begin{array}{l}3 \\
\mathbf{0}\end{array}$ & \begin{tabular}{l|}
3 \\
1
\end{tabular} & $\begin{array}{l}3 \\
2\end{array}$ & $\begin{array}{l}3 \\
3\end{array}$ & $\begin{array}{l}3 \\
4\end{array}$ & $\begin{array}{l}3 \\
5\end{array}$ & \\
\hline 1 & $\mathrm{R}$ & R & $\overline{\mathrm{R}}$ & $\bar{F}$ & $\overline{\mathrm{R}}$ & $\overline{\mathrm{E}}$ & $\bar{A}$ & $\mathrm{E}$ & $\overline{\mathrm{E}}$ & $E$ & $\mathrm{E}$ & $\mathrm{A}$ & $\mathrm{E}$ & $\mathrm{E}$ & $\mathrm{E}$ & $\mathrm{E}$ & $\mathrm{A}$ & $\mathrm{A}$ & $\mathrm{E}$ & A & 7 & $\mathrm{E}$ & 7 & . & A & $\mathrm{E}$ & $\mathrm{E}$ & $\mathrm{E}$ & $\mathrm{E}$ & $\mathrm{E}$ & $\mathrm{E}$ & $\mathrm{E}$ & $\mathrm{E}$ & $\mathrm{A}$ & $\mathrm{A}$ & A & 145 \\
\hline 2 & $\mathrm{R}$ & $\mathrm{R}$ & $\mathrm{R}$ & $\mathrm{D}$ & $\mathrm{D}$ & $\mathrm{E}$ & $\mathrm{R}$ & $\mathrm{E}$ & $E$ & $E$ & $\mathrm{~A}$ & $\mathrm{~A}$ & $\mathrm{~A}$ & $\mathrm{R}$ & $\mathrm{R}$ & $\mathrm{R}$ & $\mathrm{A}$ & $\mathrm{A}$ & $\mathrm{E}$ & $\mathrm{A}$ & $\mathrm{A}$ & E & $\mathrm{E}$ & $\vec{A}$ & & A & $\mathrm{R}$ & $\mathrm{E}$ & $\mathrm{R}$ & $\mathrm{R}$ & $\mathrm{E}$ & $\mathrm{R}$ & $\mathrm{R}$ & $\mathrm{A}$ & $\mathrm{A}$ & $\mathrm{A}$ & 136 \\
\hline 3 & $\mathrm{E}$ & $\mathrm{R}$ & $\mathrm{R}$ & $\mathrm{E}$ & $\mathrm{R}$ & $\mathrm{A}$ & $\mathrm{A}$ & $\mathrm{A}$ & $\mathrm{A}$ & $\mathrm{E}$ & $\mathrm{E}$ & $\mathrm{A}$ & $\mathrm{E}$ & $\mathrm{E}$ & $\mathrm{A}$ & $\mathrm{A}$ & $\mathrm{A}$ & $\mathrm{E}$ & $\mathrm{A}$ & $\mathrm{R}$ & $\mathrm{E}$ & $\mathrm{A}$ & $\mathrm{E}$ & $\mathrm{E}$ & 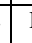 & $\mathrm{E}$ & $\mathrm{R}$ & $\mathrm{E}$ & $\mathrm{E}$ & $\mathrm{R}$ & $\mathrm{A}$ & $\mathrm{A}$ & $\mathrm{E}$ & $\mathrm{A}$ & $\mathrm{E}$ & $\mathrm{A}$ & 148 \\
\hline 4 & $\mathrm{E}$ & $\bar{A}$ & $\mathrm{R}$ & $\mathrm{E}$ & $\mathrm{E}$ & $\mathrm{E}$ & $\mathrm{R}$ & $\mathrm{E}$ & $\mathrm{E}$ & $\mathrm{E}$ & $\mathrm{A}$ & A & $\mathrm{E}$ & $\mathrm{R}$ & $\mathrm{E}$ & $\mathrm{R}$ & $\mathrm{A}$ & $\mathrm{E}$ & $\mathrm{A}$ & $\mathrm{A}$ & $\mathrm{E}$ & $\mathrm{E}$ & $\mathrm{E}$ & $A$ & & $\bar{A}$ & $\mathrm{E}$ & \begin{tabular}{l|l}
$\mathrm{R}$ \\
\end{tabular} & $\mathrm{E}$ & $\mathrm{E}$ & $\mathrm{R}$ & $\mathrm{R}$ & $\mathrm{E}$ & $\mathrm{A}$ & $\mathrm{E}$ & $\mathrm{A}$ & 143 \\
\hline 5 & $\mathrm{E}$ & $\mathrm{R}$ & $\mathrm{E}$ & $\bar{A}$ & $\mathrm{E}$ & $\mathrm{E}$ & $\mathrm{A}$ & $\mathrm{A}$ & $\mathrm{E}$ & $\mathrm{A}$ & $\mathrm{E}$ & $\mathrm{A}$ & $\mathrm{A}$ & $\mathrm{A}$ & $\mathrm{E}$ & $\mathrm{A}$ & $\mathrm{E}$ & $\mathrm{A}$ & $\mathrm{A}$ & $\mathrm{E}$ & $\mathrm{E}$ & $\mathrm{A}$ & $\mathrm{E}$ & $\mathrm{R}$ & & $\bar{E}$ & $\mathrm{E}$ & \begin{tabular}{l|l}
$\mathrm{R}$ \\
\end{tabular} & $\mathrm{E}$ & $\mathrm{E}$ & $\mathrm{E}$ & $\mathrm{E}$ & $\mathrm{E}$ & $\mathrm{A}$ & $\mathrm{E}$ & $\mathrm{A}$ & 150 \\
\hline
\end{tabular}

Fuente: Elaboración propia

Tomando en consideración las recomendaciones realizadas por los expertos, se realizaron los ajustes pertinentes. Al igual que en el instrumento anterior, aquí es de resaltar el descarte de algunos ítems y el reordenamiento de estos con base en una secuencia lógica, independientemente de la categoría a la que pertenecieran, y la eliminación de la categoría Institucional.

\section{Validación de la eficacia del contenido del instrumento para la realización de la entrevista semiestructurada}

Con el objetivo de lograr la validación de la eficacia del contenido de los ítems de la guía de entrevista del estudiante con discapacidad visual, se diseñó un instrumento con los 35 ítems de la entrevista, y se sometió a la valoración de cinco expertos, al igual que en los casos anteriores; e igualmente se acompañó de una escala de Likert del 1 al 5, donde 1 era estar totalmente en desacuerdo; 2 era estar en desacuerdo; 3 ser neutro; 4 estar de acuerdo y 5 estar totalmente de acuerdo. 


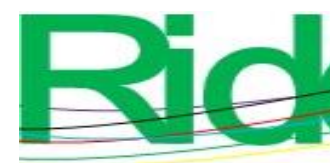

\section{Revista Iberoamericana para la
Investigación y el Desarrollo Educativo ISSN $2007-7467$}

La característica principal de este instrumento es que presentaba el objetivo de cada ítem, la categoría a la que pertenecía y el indicador correspondiente. Los expertos valoraron la eficacia del contenido de manera muy similar a la evaluación otorgada en el instrumento anterior. Los cinco expertos coincidieron en valorar la mayoría de los ítems con el mayor puntaje; los menores puntajes estuvieron destinados a los ítems correspondientes a la categoría de Institucional, bajo el argumento de la poca y prácticamente nula posibilidad de obtener esta información por parte de los estudiantes participantes (ver tabla 8).

Siguiendo estas recomendaciones, se revisó nuevamente el instrumento y se realizaron los ajustes pertinentes. Como resultado de este paso, entre otras cosas, resalta la eliminación de la categoría Institucional, la creación de un instrumento específico para este aspecto, la realización de nuevos ítems y el reordenamiento de estos con base en una secuencia lógica, con independencia de la categoría a la que pertenecieran. Como ya se mencionó, fruto de las observaciones, se elaboró un nuevo instrumento enfocado en el aspecto institucional y dirigido específicamente a los administrativos y directivos de la institución de educación superior.

Tabla 8. Validación de la eficacia del contenido de la entrevista del estudiante con discapacidad visual

\begin{tabular}{|c|c|c|c|c|c|c|c|c|c|c|c|c|c|c|c|c|c|c|c|c|c|c|c|c|c|c|c|c|c|c|c|c|c|c|}
\hline \multirow[t]{2}{*}{$\#$} & \multicolumn{33}{|c|}{ Preguntas } & \multirow[t]{2}{*}{ Total } \\
\hline & $\mathbf{1}$ & 2 & 3 & 4 & 5 & 6 & 7 & 8 & 9 & $\begin{array}{l}\mathbf{1} \\
\mathbf{0}\end{array}$ & $\begin{array}{l}\mathbf{1} \\
\mathbf{1}\end{array}$ & $\begin{array}{l}1 \\
2\end{array}$ & $\begin{array}{l}\mathbf{1} \\
3\end{array}$ & $\begin{array}{l}1 \\
4\end{array}$ & $\begin{array}{l}1 \\
5\end{array}$ & $\begin{array}{l}1 \\
6\end{array}$ & $\begin{array}{l}1 \\
7\end{array}$ & $\begin{array}{l}1 \\
8\end{array}$ & $\begin{array}{l}1 \\
9\end{array}$ & $\begin{array}{l}\mathbf{2} \\
\mathbf{0}\end{array}$ & $\begin{array}{l}2 \\
1\end{array}$ & $\begin{array}{l}2 \\
2\end{array}$ & $\begin{array}{l}2 \\
3\end{array}$ & $\begin{array}{l}2 \\
4\end{array}$ & $\begin{array}{l}2 \\
5\end{array}$ & $\begin{array}{l}2 \\
6\end{array}$ & $\begin{array}{l}2 \\
7\end{array}$ & $\begin{array}{l}2 \\
8\end{array}$ & $\begin{array}{l}2 \\
9\end{array}$ & $\begin{array}{l}3 \\
\mathbf{0}\end{array}$ & \begin{tabular}{|l}
3 \\
1
\end{tabular} & $\begin{array}{l}3 \\
2\end{array}$ & $\begin{array}{l}3 \\
3\end{array}$ & \\
\hline 1 & 1 & 3 & 1 & 2 & 2 & 4 & 4 & 5 & 4 & 4 & 5 & 5 & 3 & 3 & 5 & 5 & 5 & 4 & 3 & 5 & 5 & 5 & 5 & 2 & 2 & 4 & 3 & 5 & 4 & 4 & 5 & 5 & 5 & 127 \\
\hline 2 & 3 & 2 & 2 & 2 & 1 & 5 & 5 & 5 & 4 & 5 & 5 & 5 & 5 & 5 & 5 & 4 & 5 & 4 & 5 & 5 & 5 & 5 & 5 & 3 & 4 & 4 & 4 & 5 & 4 & 4 & 5 & 5 & 5 & 134 \\
\hline 4 & 3 & 2 & 1 & 2 & 1 & 4 & 5 & 5 & 4 & 5 & 5 & 5 & 4 & 5 & 5 & 4 & 4 & 4 & 5 & 5 & 5 & 5 & 5 & 4 & 3 & 4 & 5 & 5 & 5 & 4 & 5 & 5 & 5 & 137 \\
\hline 5 & 3 & 4 & 1 & 2 & 1 & 5 & 5 & 4 & 5 & 5 & 5 & 5 & 5 & 5 & 5 & 4 & 3 & 4 & 5 & 5 & 5 & 5 & 5 & 4 & 4 & 5 & 5 & 5 & 4 & 4 & 5 & 5 & 5 & 135 \\
\hline
\end{tabular}




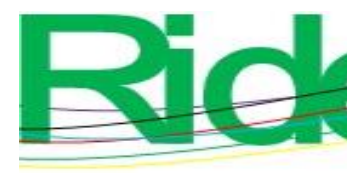

Revista Iberoamericana para la Investigación y el Desarrollo Educativo ISSN 2007 - 7467

\section{Entrevista del estudiante con discapacidad visual (versión final)}

La entrevista del estudiante con discapacidad visual recibió un tratamiento similar al de la entrevista del profesor. En este apartado se describe el instrumento final que se utilizó durante la etapa de trabajo de campo del estudio antes mencionado. La entrevista del estudiante con discapacidad visual consta de una presentación que incluye el objetivo de la entrevista y los datos generales del entrevistado. La sección de preguntas consta de cinco situaciones que tienen como objetivo ubicar al alumno de lenguas extranjeras con discapacidad visual en un momento y situación específicos de su experiencia en el aprendizaje de lenguas extranjeras.

Cada una de estas situaciones incluye preguntas relacionadas con las dos categorías que desarrolla la entrevista (Educativo y Social). La primera situación plantea lo siguiente: "Pensemos en uno de tus cursos de lenguas extranjeras". A través de esta situación se busca obtener información relacionada con las estrategias de aprendizaje empleadas por el discente durante un curso en particular. Las preguntas que se contemplaron en esta situación pertenecen a la categoría Educativo.

La segunda situación que se consideró fue la siguiente: "Pensemos en tus primeras clases de lengua extranjera". Aquí se buscaba ubicar al estudiante con discapacidad visual en el momento en el que tenía su primer contacto tanto con el profesor como con sus pares y el aula de clase. Las preguntas que se consideraron en esta situación están igualmente relacionadas con la categoría Educativo.

Con el objetivo de ubicar al estudiante en el contexto de la enseñanza y el aprendizaje de lenguas extranjeras en el nivel superior se plantea la tercera situación: "Pensemos en los cursos de lenguas extranjeras que has cursado en la universidad".

Con la intención de obtener información sobre los conocimientos del entrevistado en relación con la lengua extranjera, se plantea la siguiente situación: "Ahora que ya concluyeron tus clases de lenguas extranjeras..." acompañada de diversos ítems.

La última situación que se plantea en esta entrevista fue la siguiente: "Pensemos en aquellas sesiones en las que tu profesor desarrollaba alguna actividad de lecto-escritura". Específicamente, esta situación presenta ítems relacionados con la categoría de lo Social, a través de la cual se busca obtener información sobre las relaciones interpersonales entre el estudiante con discapacidad, el profesor y el resto del alumnado. 


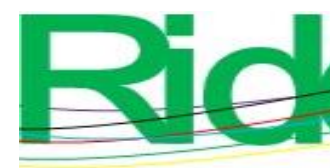

Revista Iberoamericana para la Investigación y el Desarrollo Educativo ISSN 2007 - 7467

\section{Entrevista del administrador}

Con base en la experiencia de pilotaje y validación de los dos instrumentos previamente presentados, se elaboró la entrevista al administrador (sugerida por los expertos durante el proceso de validación), la cual no requirió del proceso de validación antes presentado en virtud de que ya habían sido valoradas las situaciones y los ítems que la conformaban en los instrumentos previos.

La entrevista para el administrador consiste en una entrevista semilibre (Acevedo y López, 2008) en la que el participante debía responder sobre cuestiones relacionadas solo con la categoría llamada Institucional, consecuencia de la validación de los instrumentos anteriores. La investigadora planteó preguntas pertinentes relacionadas con su práctica educativa en los aspectos antes señalados.

\section{Descripción del instrumento}

La guía de entrevista inicial del coordinador contenía la razón social de las instituciones involucradas, el objetivo de la entrevista y los datos de identificación del coordinador, tales como nombre, edad, asignatura y ciclo en el que cursó dicha asignatura. El instrumento contó con 17 preguntas.

Las entrevistas se realizaron en horarios diversos, según la disponibilidad de los profesores, administradores y estudiantes con discapacidad visual. En su mayoría fueron realizadas por la mañana en la sala de maestros y la sala de espera de la institución participante. El instrumento empleado para tales efectos fue una grabadora de voz digital.

Para el caso de cada instrumento, una vez que se reestructuraron a partir de las recomendaciones de los expertos, se convocó a cada uno de ellos y se les presentó la guía de entrevista, la cual revisaron y discutieron. Como producto de este encuentro, se obtuvo la última retroalimentación y se elaboró la versión final de la entrevista. 


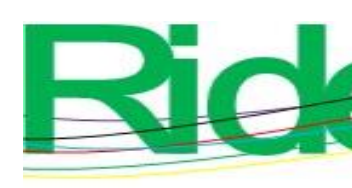

Revista Iberoamericana para la Investigación y el Desarrollo Educativo ISSN $2007-7467$

\section{Discusión}

El proceso validación de entrevistas semiestructuradas que se presentó en este documento permitió conocer algunos aspectos sobre las prácticas educativas que convergen en los procesos de enseñar y aprender lenguas extranjeras en estudiantes con discapacidad visual. Resaltamos la importancia en estos procesos del rol desempeñado por los expertos, ya que, sin su participación seria y responsable, los productos (tres entrevistas) no se hubieran logrado.

Cada paso del proceso tuvo implicaciones metodológicas que generaron instrumentos elaborados acorde a una realidad en específico y a las necesidades involucradas en esta. En relación con el primer paso, este fue posible a través de un buen anclaje entre el abordaje teórico del estudio y los elementos metodológicos requeridos para la estructuración de una entrevista semiestructurada. Durante el paso dos, se experimentó el proceso de la implementación de una entrevista, donde se involucraron aspectos esenciales del protocolo de entrevistas que se enfocan a cuestiones como el espacio y el tiempo. El paso tres dejó en claro la cuestión de las herramientas y elementos necesarios para la consulta a expertos. Es de resaltar el hecho de que un instrumento debe de ser analizado tomando en cuenta el contexto investigativo y metodológico que le dio origen. Los pasos cuatro y cinco hacen énfasis en la importancia de la revisión constante y permanente del instrumento antes de ser aplicado formalmente en el estudio.

Vinculados con la validez de entrevistas, estudios previos han diseñado, en un primer momento, el instrumento con apoyo de expertos y, posteriormente, lo han validado mediante una prueba piloto (Mediavilla y García, 2013); otros se han enfocado en el análisis de ítems de las entrevistas o han trabajado validaciones de entrevistas codificadas gracias a las cuales han obtenido buenos resultados en sus respectivos procesos de validación (Robles, Robles, Giménez y Abad, 2016). El estudio que aquí se presenta se caracterizó por una doble valoración metodológica, de tipo de contenido y de eficacia de contenido, además de presentar una doble valoración, de situaciones y de ítems del instrumento.

En relación con los resultados de la entrevista en el estudio sobre las prácticas educativas que convergen en la enseñanza y el aprendizaje de lenguas extranjeras a estudiantes con discapacidad visual en el nivel superior, estos se pueden resumir de la siguiente manera. Al respecto de la enseñanza de lenguas extranjeras a estudiantes con discapacidad, los actores indicaron que no en todos los casos existe una preparación 


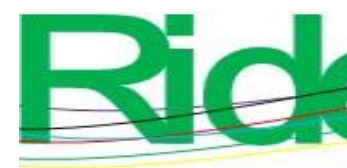

Revista Iberoamericana para la
Investigación y el Desarrollo Educativo
ISSN $2007-7467$

específica para la atención a esta población, lo cual dificulta la enseñanza de la lengua extranjera, y que en aquellos casos en los que la capacitación sí se ha llevado a cabo, esta no se proyecta en el aula, lo que abre un gran vacío en los procesos de formación.

Por lo anterior, algunos profesores han optado por diseñar sus propias estrategias de enseñanza a estos alumnos, las cuales no han sido totalmente correctas, a decir de los estudiantes, pues les han impedido principalmente desarrollar la competencia de escritura. Se señaló que, si bien la institución se ha encargado en los últimos años de equipar sus aulas para proporcionarles un mejor servicio a estos estudiantes y ha adquirido equipo tecnológico y máquinas de impresión en braille, esto no ha sido funcional en virtud de la falta de socialización de estas acciones y la falta de capacitación para sus profesores. Con respecto al aprendizaje, los estudiantes señalan que cuentan con estrategias y herramientas específicas para el desarrollo de las cuatro habilidades de la lengua (receptivas: leer y escuchar; productivas: hablar y escribir), sin embargo, sus profesores lo ignoran; al hacer esto, develan la falta de comunicación entre estos actores.

\section{Conclusiones}

Desde un plano metodológico, las guías de entrevista fueron diseñadas por el investigador, con apoyo de un grupo de expertos; tras la obtención de un alto consenso y una alta fiabilidad (alfa de Cronbach > 0.80), fueron sometidas a una prueba piloto y, posteriormente, validadas.

En el presente artículo hemos expuesto una experiencia que ha hecho evidente la importancia de la planeación de las estructuras metodológicas en todo proceso de investigación. La riqueza de que, a consecuencia de la aplicación de las estructuras metodológicas, sea necesario reformular preguntas para redireccionar la interpretación es ampliamente valorada. Este documento muestra la importancia de una adecuada formulación de objetivos y procedimientos que oriente a los expertos durante el proceso de validación y, con ello, eviten, entre otros elementos, la carga de subjetividad en sus respuestas. 


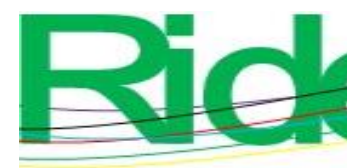

Revista Iberoamericana para la Investigación y el Desarrollo Educativo ISSN 2007 - 7467

\section{Limitaciones y fortalezas del estudio}

Este estudio sobre validación de entrevistas semiestructuradas cuenta tanto con limitaciones como con fortalezas; ambas han quedado en evidencia durante el desarrollo de este trabajo. Entre las limitaciones, destaca la escasez de estudios sobre validación de entrevistas semiestructuradas que provean de referentes teóricos y metodológicos que permitan un mayor sustento en estos procesos y la falta de coordinación entre todos los actores del proceso de validación. Entre sus fortalezas, sobresale la ética profesional que caracterizó a los expertos que participaron en el estudio, vertida en pro del desarrollo de investigaciones científicas, y el seguimiento puntual de quienes coordinaron el proceso de validación, lo que permitió la pronta identificación de problemas.

\section{Referencias}

Acevedo, A. y López, A. (2008). El proceso de la entrevista: Conceptos y modelos. México: Limusa.

Deng, M. and Guo, L. (2007). Local special education administrators' Understanding of Inclusive Education in China. International Journal of Educational Development, 27(6), 697-707.

Dorantes, J. A., Hernández, J. S. y Tobón, S. (2016). Juicio de expertos para la validación de un instrumento de medición del síndrome de burnout en la docencia. Ra Ximhai, 12(6), 327-346.

Galicia, L. A., Balderrama, J. A. y Edel, R. (2017). Validez de contenido por juicio de expertos: propuesta de una herramienta virtual. Apertura, 9(2), 42-53. Recuperado de http://www.scielo.org.mx/pdf/apertura/v9n2/2007-1094-apertura-9-0200042.pdf.

Ghergut, A. (2010). Analysis of inclusive education in Romania: Results from a survey conducted among teachers. Procedia - Social and Behavioral Sciences, 5, 711-715.

Ginsburg, H. P. (1997). Entering the Child's Mind. The Clinical Interview in Psychological Research and Practice. New York, United States: Cambridge University Press.

Malinen, O., Savolainen, H. and Xu, J. (2012). Beijing-in-service teachers' self-efficacy and attitudes towards inclusive education. Teaching and Teacher Education, 28(4), 526534. 

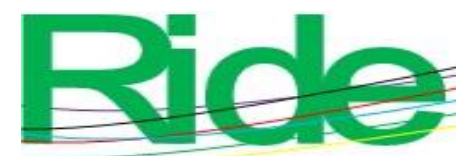

Revista Iberoamericana para la Investigación y el Desarrollo Educativo

ISSN 2007 - 7467

Mediavilla, L. y García, J. M. (2013). Diseño, creación y validación de una entrevista para obtener datos biográficos, de carácter deportivo-militar, de los militares que participaron en unos juegos olímpicos. Journal of Sport and Health Research, 5(2), 157-166.

Mustafa, B. (2011). Inclusion: something more than sitting together. Paper presented at the International Centre for Innovation in Education (ICIE-2011) Conference. Istanbul, July 6-9, 2011.

Organización de las Naciones Unidas para la Educación, la Ciencia y la Cultura [Unesco]. (2016). Educación 2030: Declaración de Incheon y Marco de Acción para la realización del Objetivo de Desarrollo Sostenible 4. Organización de las Naciones Unidas para la Educación, la Ciencia y la Cultura.

Plancarte, P. A. (2017) Inclusión educativa y cultura inclusiva. Revista Nacional e Internacional de Educación Inclusiva, 10(2), 213-226.

Robles, A., Robles, J., Giménez, F. J. y Abad, M. T. (2016) Validación de una entrevista para estudiar el proceso formativo de judokas de élite. Revista Internacional de Medicina y Ciencias de la Actividad Física y el Deporte, 16(64), 723-738 Recuperado de http://cdeporte.rediris.es/revista/revista64/artvalidacion757.htm.

Shevlin, M., Kenny, M. and Me Neela, E. (2004). Access routes to higher education for young people with disabilities: A question of chance? Irish Educational Studies, 23(2), 37-53.

Unianua, E. M. (2012). Teachers' attitudes towards inclusive education. Procedia - Social and Behavioral Sciences, 33, 900-904. 


\begin{tabular}{|c|c|}
\hline Rol de Contribución & Autor (es) \\
\hline Conceptualización & Veronika de la Cruz Villegas (principal) \\
\hline Metodología & $\begin{array}{l}\text { Veronika de la Cruz Villegas (igual) } \\
\text { Eduardo Jesús Gordillo Fuentes }\end{array}$ \\
\hline Software & NO APLICA \\
\hline Validación & $\begin{array}{l}\text { Veronika de la Cruz Villegas (igual) } \\
\text { Eduardo Jesús Gordillo Fuentes }\end{array}$ \\
\hline Análisis Formal & $\begin{array}{l}\text { Veronika de la Cruz Villegas (igual) } \\
\text { Eduardo Jesús Gordillo Fuentes }\end{array}$ \\
\hline Investigación & $\begin{array}{l}\text { Dra. Veronika de la Cruz Villegas (igual) } \\
\text { Dr. Eduardo Jesús Gordillo Fuentes }\end{array}$ \\
\hline Recursos & $\begin{array}{l}\text { Dra. Veronika de la Cruz Villegas (igual) } \\
\text { Dr. Eduardo Jesús Gordillo Fuentes }\end{array}$ \\
\hline Curación de datos & Dr. Eduardo Jesús Gordillo Fuentes \\
\hline $\begin{array}{l}\text { Escritura - Preparación del } \\
\text { borrador original }\end{array}$ & $\begin{array}{l}\text { Veronika de la Cruz Villegas (principal) } \\
\text { Eduardo Jesús Gordillo Fuentes (que apoya) }\end{array}$ \\
\hline $\begin{array}{l}\text { Escritura - Revisión y } \\
\text { edición }\end{array}$ & $\begin{array}{l}\text { Veronika de la Cruz Villegas (igual) } \\
\text { Eduardo Jesús Gordillo Fuentes }\end{array}$ \\
\hline Visualización & $\begin{array}{l}\text { Veronika de la Cruz Villegas (igual) } \\
\text { Eduardo Jesús Gordillo Fuentes }\end{array}$ \\
\hline Supervisión & $\begin{array}{l}\text { Veronika de la Cruz Villegas (principal) } \\
\text { Eduardo Jesús Gordillo Fuentes ( que apoya) }\end{array}$ \\
\hline $\begin{array}{l}\text { Administración de } \\
\text { Proyectos }\end{array}$ & $\begin{array}{l}\text { Veronika de la Cruz Villegas (principal) } \\
\text { Eduardo Jesús Gordillo Fuentes (que apoya) }\end{array}$ \\
\hline Adquisición de fondos & $\begin{array}{l}\text { Veronika de la Cruz Villegas (igual) } \\
\text { Eduardo Jesús Gordillo Fuentes }\end{array}$ \\
\hline
\end{tabular}

